01,11

\title{
Спинодальный распад сплава с сильной концентрационной зависимостью коэффициента взаимной диффузии
}

\author{
(C) И.К. Разумов
}

Институт фризики металлов им. М.Н. Михеева УрО РАН,

Екатеринбург, Росси

E-mail: rik@imp.uran.ru

Поступила в Редакцию 15 июня 2021 г.

В окончательной редакции 15 июня 2021 г.

Принята к публикации 9 сентября 2021 г.

Показано, что кинетика распада сплава с сильной концентрационной зависимостью коэффициента взаимной диффузии качественно отличается от широко известной кинетики спинодального распада, поскольку включает формирование метастабильных (в кинетическом смысле) выделений промежуточного состава. Концентрационная зависимость коэффициента взаимной диффузии обусловлена, в частности, различием коэффициентов примесной диффузии, а также коэффициентов самодиффузии компонентов, и усиливается с понижением температуры. В расчетах используется выражение для потока атомов компонента в отсутствие течения вещества, ранее полученное в рамках кинетической теории диффузии по вакансиям („метод дырочного газа“).

Ключевые слова: спинодальный распад, коэффициент взаимной диффузии, выделения промежуточного состава.

DOI: $10.21883 /$ FTT.2022.01.51826.143

\section{1. Введение}

В теории фазовых превращений спинодальным распадом (Spinodal Decomposition, SD) называют формирование концентрационных неоднородностей и последующее расслоение на фазы в результате химического взаимодействия атомов в первоначально однородном сплаве $[1,2]$. Классическая кинетика SD включает следующие стадии: волновая (нарастание длинноволновых флуктуаций состава), коалесценция волн и испарение-конденсация капель. Волновая стадия распада была впервые описана в работах Кана [3,4]. Стадия коалесценции волн исследовалась, например, в работах $[5,6]$. На стадии испарения-конденсации крупные выделения растут за счет мелких, подчиняясь кинетике Лившица-Слезова [7]. Кристаллическая решетка сплава в процессе SD остается неизменной либо перестраивается на поздних стадиях.

В большинстве работ особое внимание уделяется влиянию термодинамических факторов (вид функции плотности свободной энергии, включая непарный характер межатомных взаимодействий или возможность упорядочения, дальнодействия, присутствие градиентных вкладов в функционале свободной энергии, роль тепловых флуктуаций, скорость охлаждения и т.д.) на кинетику SD, в то время как коэффициент диффузии для простоты заменяется константой. В этом случае характерная диффузионная длина $L=\left(D^{*} t\right)^{1 / 2}$ не зависит от координат, поэтому на границе возникшего выделения быстро достигается локальное термодинамическое равновесие, и концентрация в объеме растущего выделения близка к равновесному значению. Однако, следует ожидать изменения кинетики превращения, если коэффициенты диффузии в матрице и в области формирующегося выделения существенно различаются, поскольку в этом случае рост выделения может происходить в условиях, когда локальное равновесие на его границе не достигнуто.

Как известно из теории диффузии [1], скорость перераспределения атомов в бинарном сплаве контролируется коэффициентом взаимной диффузии $D=D_{A}^{*} c_{A}+D_{B}^{*} c_{B}$, где $D_{A(B)}^{*}-$ собственные (парциальные) коэффициенты диффузии, $c_{A(B)}$ - атомные концентрации компонентов. При этом собственные коэффициенты диффузии часто считают близкими коэффициентам диффузии меченых атомов $D_{A(B)}$, что в общем случае трудно считать оправданным. Действительно, в отсутствие течения вещества (потока вакансий) из теории диффузии следует $D=D_{A}^{*}=D_{B}^{*}[1]$. Между тем, можно физически обеспечить это условие, запретив поток вещества, например, поместив сплав в сосуд с неподвижными стенками. Поскольку коэффициенты диффузии меченых атомов $D_{A(B)}$ являются неизменными микроскопическими параметрами, отсюда следует $D_{A(B)} \neq D_{A(B)}^{*}$. Поэтому выражение $D \approx D_{A} c_{A}+D_{B} c_{B}$ может быть справедливо только в том случае, когда разрешено течение вещества (эффект Киркендалла [8]). В свою очередь, течение вещества предполагает наличие источников и стоков неравновесных вакансий в диффузионной зоне, в качестве которых могут выступать различные дефекты решетки [9]. Такая ситуация наиболее характерна при диффузионном перемешивании хорошо смешиваемых металлов приведенных в контакт, и менее ожидаема на 
Значения коэффициентов примесной диффузии и самодиффузии в некоторых бинарных сплавах на основании справочных данных [15]

\begin{tabular}{c|c|c|c|c|c}
\hline Сплав АВ & $T, \mathrm{~K}$ & $\begin{array}{c}D_{A}\left(c_{A} \rightarrow 0\right), \\
\mathrm{m}^{2} / \mathrm{s}\end{array}$ & $\begin{array}{c}D_{B}\left(c_{B} \rightarrow 0\right), \\
\mathrm{m}^{2} / \mathrm{s}\end{array}$ & $\begin{array}{c}D_{A}\left(c_{A}=1\right), \\
\mathrm{m}^{2} / \mathrm{s}\end{array}$ & $\begin{array}{c}D_{B}\left(c_{B}=1\right), \\
\mathrm{m}^{2} / \mathrm{s}\end{array}$ \\
\hline ГЦК-FeCu & 1200 & $5 \cdot 10^{-14}$ & $2.5 \cdot 10^{-17}$ & $1.2 \cdot 10^{-17}$ & $5.5 \cdot 10^{-14}$ \\
ГЦК-AgCu & 500 & $2.5 \cdot 10^{-25}$ & $2.1 \cdot 10^{-23}$ & $7.3 \cdot 10^{-24}$ & $1.8 \cdot 10^{-26}$ \\
ОЦК- $\mathrm{FeCr}$ & 750 & $3.4 \cdot 10^{-28}$ & $8.7 \cdot 10^{-22}$ & $2.9 \cdot 10^{-22}$ & $2 \cdot 10^{-32}$
\end{tabular}

начальных стадиях спинодального распада из однородного состояния.

В работах $[10,11,12]$ в рамках кинетической теории диффузии по вакансиям („метод дырочного газа“ [13]) были получены выражения для потоков атомов при наличии градиентов концентрации вакансий. В этом подходе, в случае пренебрежимо малого потока вакансий $\left(\left|J_{V}\right| \ll\left|J_{A(B)}\right|\right)$, в вакансионной подсистеме достигается локальное равновесие, соответствующее локальным концентрациям компонентов, а выражение для коэффициента взаимной диффузии имеет вид: $D=D_{A} D_{B}\left(1-\Psi c_{A} c_{B}\right) /\left(D_{A} c_{A}+D_{B} c_{B}\right)$, причем в случае идеального твердого раствора $\Psi=0$. Аналогичное по сути выражение было получено в работе [14]. Можно видеть, что в предельных случаях сильно разбавленных твердых растворов коэффициент взаимной диффузии принимает значения $D=D_{A}\left(c_{A} \rightarrow 0\right)$ или $D=D_{B}\left(c_{B} \rightarrow 0\right)$ соответственно (далее для краткости $\left.D_{A}\left(c_{A} \rightarrow 0\right) \equiv D_{A}^{0}, D_{B}\left(c_{B} \rightarrow 0\right) \equiv D_{B}^{0}\right)$. Следовательно, в процессе SD формируются области, в которых коэффициент взаимной диффузии (и вместе с ним собственные коэффициенты диффузии $\left.D_{A}^{*}, D_{B}^{*}\right)$ меняется от значений близких к $D_{A}^{0}$ (коэффициент диффузии примеси А в матрице В) до значений близких к $D_{B}^{0}$ (коэффициент диффузии примеси В в матрице А).

В таблице приведены значения коэффициентов примесной диффузии $D_{A}^{0}, D_{B}^{0}$, а также коэффициентов самодиффузии $D_{A}^{1}, D_{B}^{1}$ для нескольких сплавов претерпевающих SD, на основании справочных данных [15]. Можно видеть, что при выбранных температурах значения $D_{A}^{0}$, $D_{B}^{0}$, различаются на несколько порядков. При этом коэффициент диффузии примеси в матрице обычно имеет величину того же порядка, что и коэффициент самодиффузии атомов матрицы. В силу того, что температурные зависимости коэффициентов диффузии подчинены закону Аррениуса, с понижением температуры отношение $D_{A} / D_{B}$ должно изменяться экспоненциально. Во всех выбранных случаях $\mathrm{SD}$ реализуется без перестройки кристаллической решетки. В других сплавах возможная перестройка решетки в процессе SD создает дополнительные возможности для зависимости $D$ от локального состояния сплава в процессе превращения.

Приведенные данные позволяют предполагать, что ситуация, когда рост выделений в процессе SD происходит в условиях далеких от достижения локального равновесия вблизи межфазной границы, является вполне возможной. Далее предлагается простая модель, позволяющая исследовать кинетику SD при наличии концентрационной зависимости коэффициента взаимной диффузии $D$, и обсуждаются характерные сценарии, полученные в результате численного решения предложенных уравнений.

\section{2. Формулировка модели}

Согласно работам $[10,11,12]$, выражение для потока атомов компонента А в бинарном сплаве при запрете течения вещества (т.е. в пределе малого потока вакансий, $\left.\left|J_{V}\right| \ll\left|J_{A(B)}\right|\right)$ может быть записано в виде

$\mathbf{J}_{\mathrm{A}}=-\frac{D_{A} D_{B}}{D_{A} c_{A}+D_{B} c_{B}}\left[\left(1-\Psi c_{A} c_{B}\right) \nabla c_{A}-R^{2} \Psi c_{A} c_{B} \nabla \Delta c_{A}\right]$,

где $D_{i}=c_{V} \omega_{i}$ - коэффициенты диффузии меченых атомов, $c_{V}$ - концентрация вакансий, $\omega_{i}-$ диффузионные подвижности, $c_{A(B)}$ - атомные концентрации компонентов связанные условием $c_{A}+c_{B}=1, \Psi=-2 v /(k T)-$ безразмерная энергия смешения, $R$ - малый параметр определяющий ширину межфазной границы. Коэффициенты диффузии $D_{i}$ подчиняются уравнению Аррениуса, в котором энергия активации линейно зависит от концентрации компонента

$$
D_{i}=D_{0 i}^{0} \exp \left[-\frac{U_{0}+U_{1} c_{i}}{k T}\right] .
$$

Учитывая, что из экспериментов известны коэффициенты примесной диффузии $D_{i}\left(c_{i} \rightarrow 0\right) \equiv D_{i}^{0}$ и коэффициенты самодиффузии $D_{i}\left(c_{i}=1\right) \equiv D_{i}^{1}$, перепишем (2) в эквивалентной форме

$$
D_{i}=D_{i}^{0}\left[D_{i}^{1} / D_{i}^{0}\right]^{c_{i}} .
$$

Для описания эволюции сплава используем уравнение непрерывности

$$
\frac{\partial c_{A}}{\partial t}=-\nabla \mathbf{J}_{\mathrm{A}}
$$

\section{3. Сценарии спинодального распада}

Система уравнений (1)-(4) решалась численно на 2D-области с использованием двухслойной явной конечно-разностной схемы, с безразмерным временем 


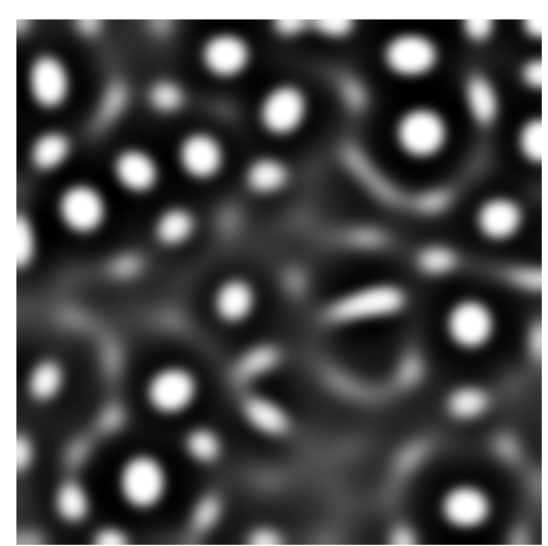

$\tau=0.012$

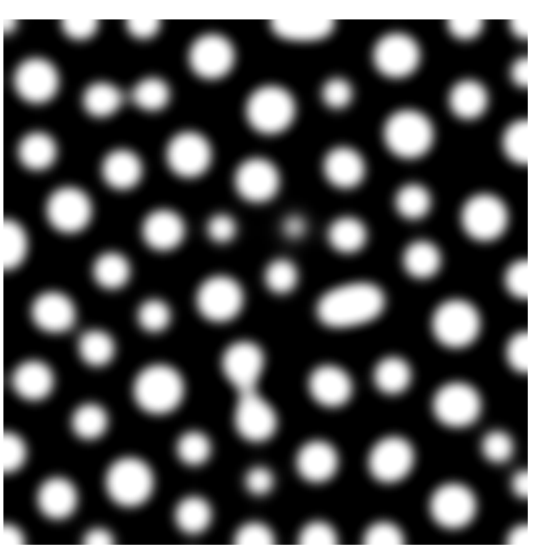

0.022

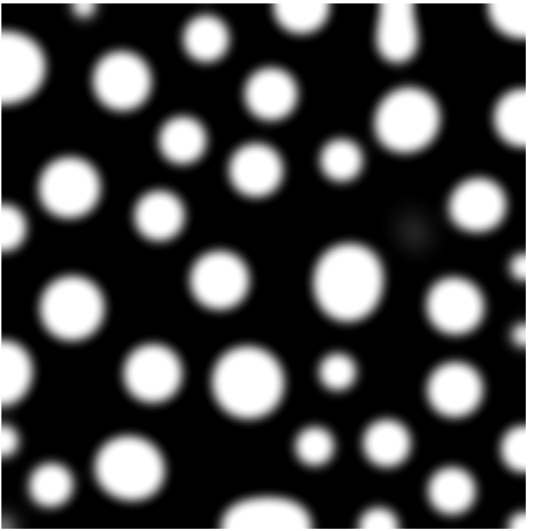

0.100

Рис. 1. Кинетика спинодального распада при $c_{A 0}=0.3, \Psi=6.5, D_{A}^{0}=D_{A}^{1}=D_{B}^{0}=D_{B}^{1}, R / L=7 \cdot 10^{-3}$.

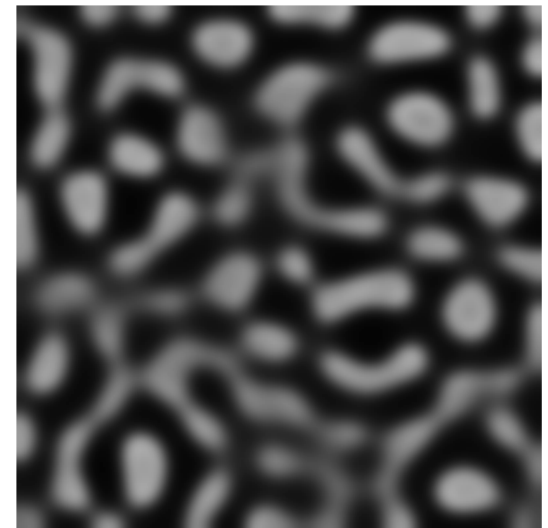

$\tau=0.22$

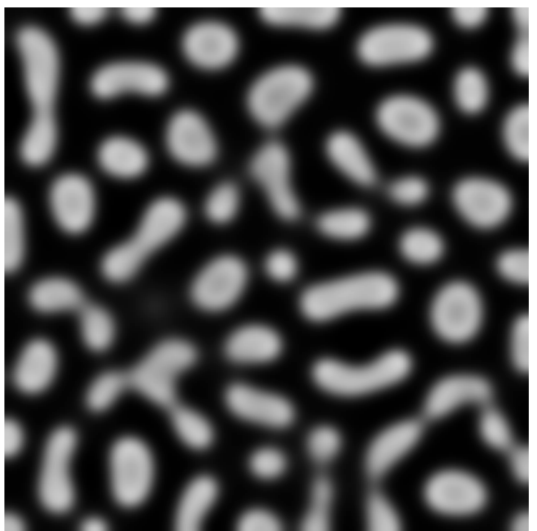

0.29

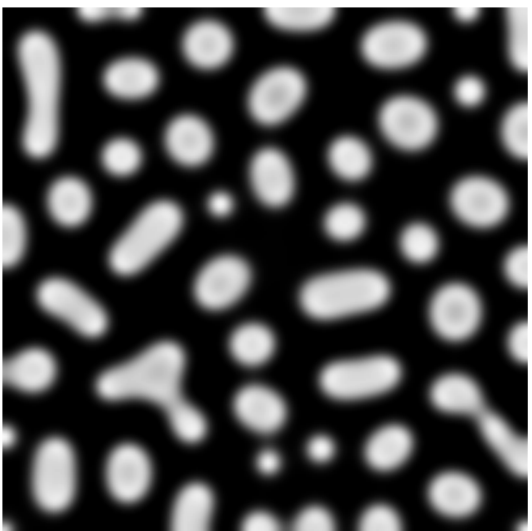

0.50

Рис. 2. Кинетика спинодального распада при $c_{A 0}=0.3, \Psi=6.5, D_{A}^{0} / D_{B}^{0}=D_{B}^{1} / D_{A}^{1}=10^{4}, D_{A}^{0} / D_{B}^{1}=1, R / L=7 \cdot 10^{-3}$.

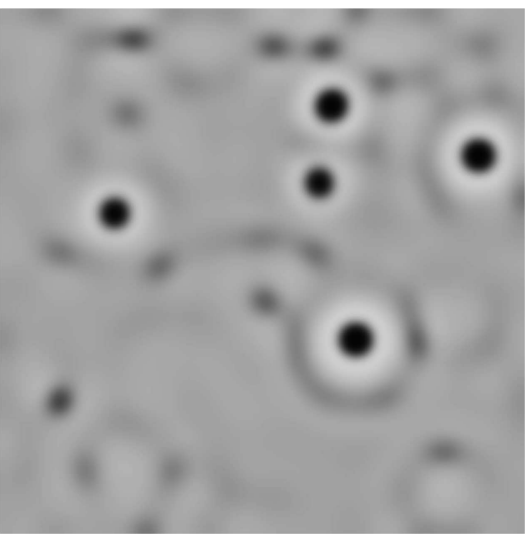

$\tau=5.4$

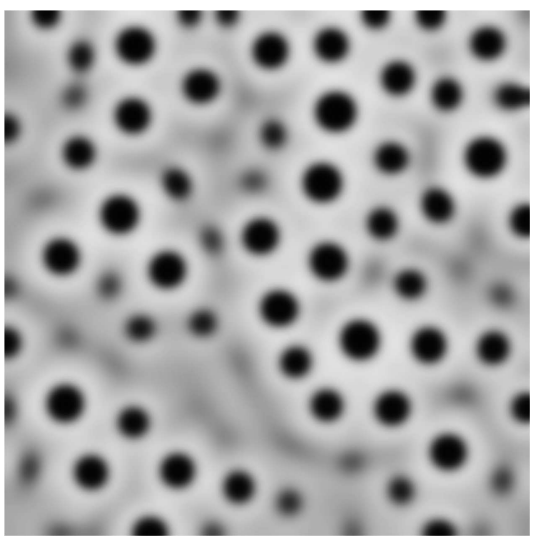

7.6

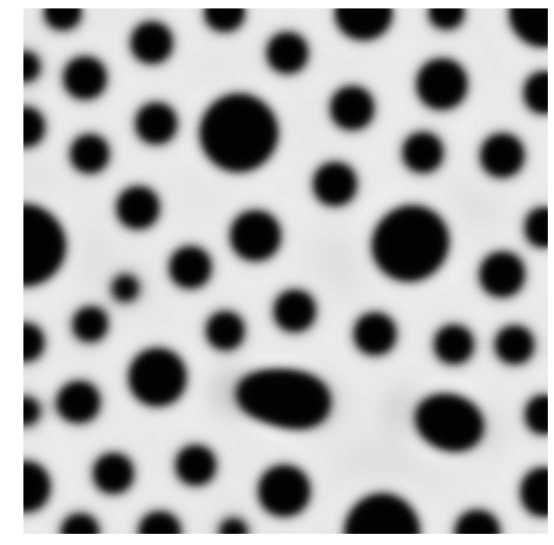

22.0

Рис. 3. Кинетика спинодального распада при $c_{A 0}=0.7$, остальные параметры аналогичны рис. 2.

$\tau=t D_{A}^{0} / L^{2}$ и координатами $x / L, y / L(L-$ размер расчетной области). Начальное состояние выбиралось однородным со средней концентрацией $c_{A 0}$, с малыми гауссовыми флуктуациями состава. Использовались „зеркально-симметричные“ граничные условия, означа- ющие отсутствие потоков компонентов через границы квадратной области.

На рис. 1-4 представлены результаты расчетов при различном выборе коэффициентов диффузии. Уровни концентрации компонента А обозначаются градациями 


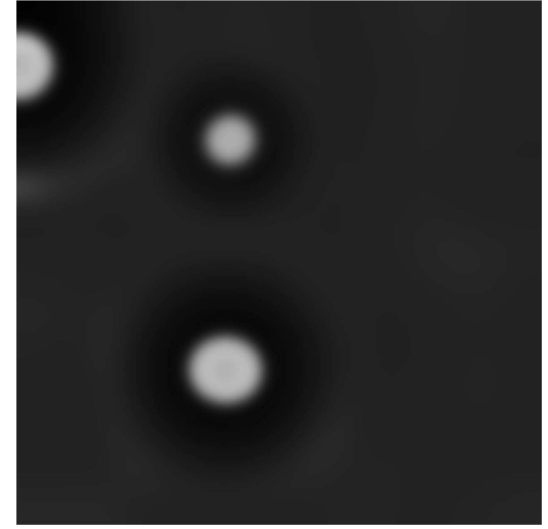

$\tau=0.72$

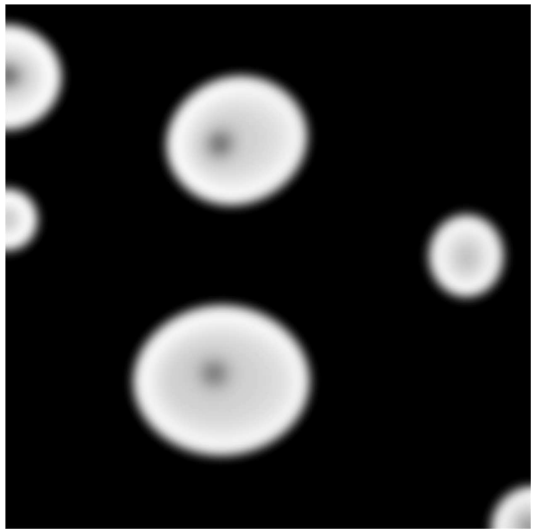

2.10

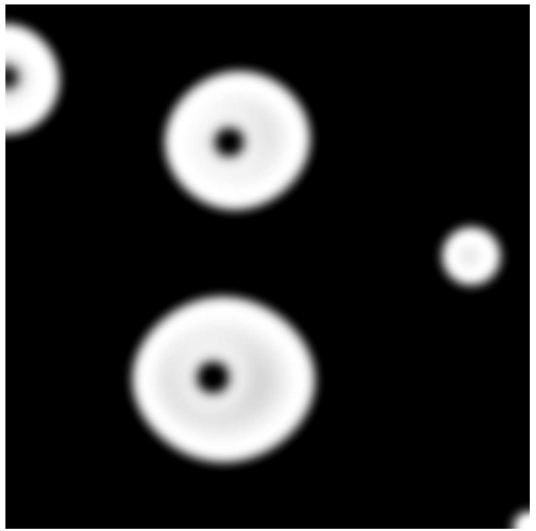

5.00

Рис. 4. Кинетика спинодального распада при $c_{A 0}=0.2$, остальные параметры аналогичны рис. 2.
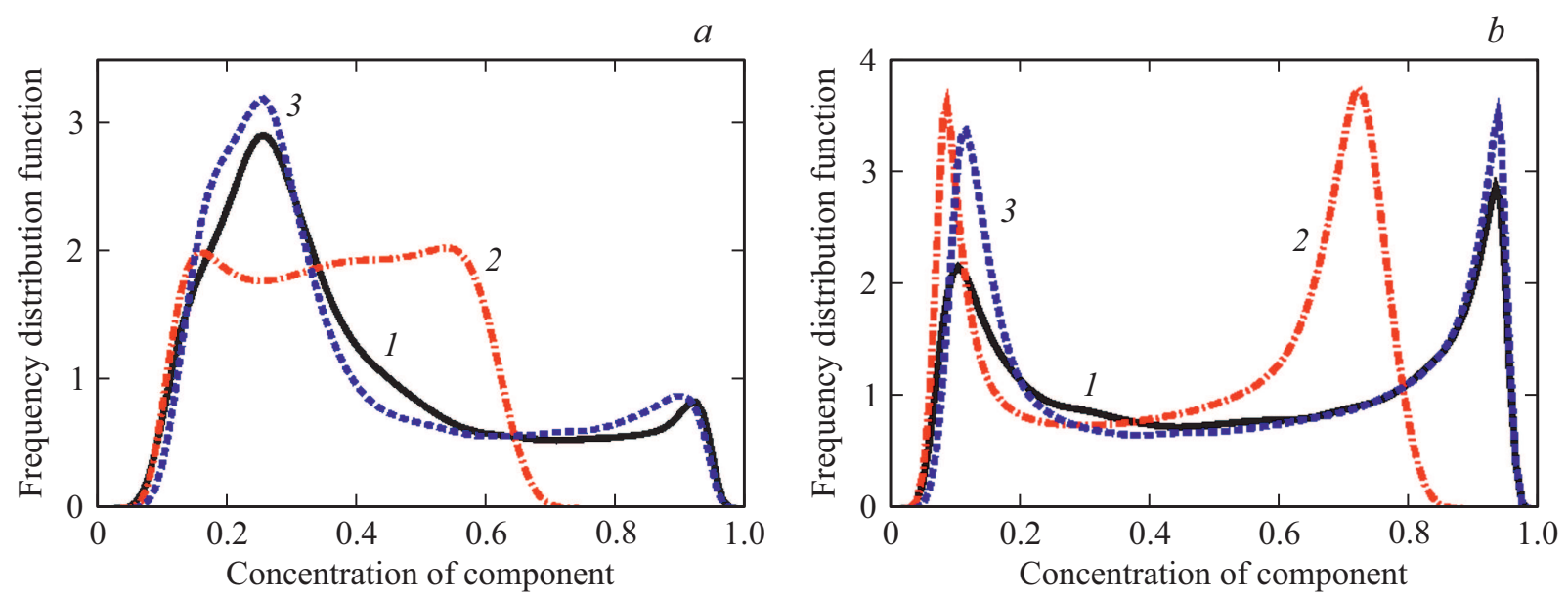

Рис. 5. Функция распределения по концентрациям компонента образующего выделения при параметрах соответствующих рис. 1 (кривая 1), рис. 2 (кривая 2), рис. 3 (кривая 3) в момент времени соответствующий достижению степени распада 0.3 (a), 0.5 (b).

серого цвета (черный цвет соответствует $c_{A}=0$, белый $\left.-c_{A}=1\right)$. Заметные качественные отличия от классической кинетики SD (рис. 1) возникают, если отношение $D_{A}^{0} / D_{B}^{0}$ составляет $10^{3} \ldots 10^{4}$ или более (рис. 2-4).

Если исходное однородное состояние соответствует высоким значениям $D\left(c_{A}\right)$, флуктуации состава нарастают гомогенно по всему объему, и к моменту завершения волновой стадии распада возникает микроструктура сформированная выделениями неправильной формы, промежуточного состава (рис. 2). Дальнейшая эволюция сводится к огрублению микроструктуры и удалению избыточной примеси из объема выделений.

Если исходное однородное состояние соответствует низким значениям $D\left(c_{A}\right)$, то после значительной выдержки возникает дисперсная структура, сформированная каплями правильной формы, имеющими состав близкий к равновесному и несколько меньший размер, по сравнению со структурой, возникающей при $D=$ const (рис. 3). При этом матрица остается пересыщена компонентом А даже после формирования выделений, а тенденция автокатализа выделений на волновой стадии (когда возникшие выделения приводят к появлению новых выделений в своей окрестности) более заметна, чем в случае $D=$ const, где волновая стадия развивается относительно равномерно по объему. Стадия испаренияконденсации капель оказывается подавленной, потому что развитие распада сопровождается снижением скорости диффузии в матрице.

При понижении средней концентрации выделения становятся более редкими (ср. рис. 4 и 2), так что в их объеме оказывается захваченным большее количество примеси. Эта примесь не успевает диффундировать из объема выделений в матрицу даже на развитых стадиях распада, что в итоге приводит к возникновению вторичных выделений внутри первичных (рис. 4).

Для анализа кинетики распада удобно ввести интегральную степень распада

$$
S_{d e c}=\left(2 c_{A 0}\left(1-c_{A 0}\right)\right)^{-1} \int\left|c_{A}(\mathbf{r})-c_{A 0}\right| d \mathbf{r},
$$

где $c_{A 0}-$ средняя по образцу концентрации компонента А. Величина $S_{d e c}$ может принимать значения от 0 
(в однородном состоянии) до 1 (при распаде на чистые компоненты).

Кроме того, удобно ввести функцию распределения компонента по концентрациям. Значение этой функции при заданной концентрации соответствует плотности вероятности того, что случайно выбранный атом данного компонента находится в области пространства с соответствующей локальной концентрацией. В начале эволюции эта функция имеет один максимум, соответствующий средней концентрации компонента. По завершении распада она имеет два максимума, соответствующие равновесным пределам растворимости.

На рис. 5 представлены графики функции распределения компонента по его концентрациям при достижении одинаковой степени распада $S_{d e c}$ в трех представленных выше сценариях кинетики (рис. 1-3). При этом рассматривается компонент образующий выделения, т.е. компонент А в первых двух сценариях, и компонент В в последнем случае. Присутствие выделений промежуточного состава в сценарии представленном на рис. 2 выражено тем фактом, что соответствующая функция распределения (кривая 2 на рис. 5) имеет максимум в области промежуточных концентраций $0.6 \ldots 0.8$. В отличие от этого, в случае классического SD второй максимум функции появляется сразу вблизи равновесного предела растворимости (см. кривую 1 на рис. 5). Пересыщенность матрицы в сценарии представленном на рис. 3 выражена тем фактом, что на развитых стадиях распада максимум функции распределения (кривая 3 на рис. 5)при небольших концентрациях $(\sim 0.1)$ расположен несколько правее соответствующих максимумов для других сценариев.

\section{4. Обсуждение}

Как следует из вышесказанного, формирование выделений промежуточного состава в процессе распада сплава наиболее ожидаемо при пониженных температурах. При этом средние концентрации компонентов должны быть сопоставимы, т.е. речь идет прежде всего о концентрированных твердых растворах. В этом случае характерная диффузионная длина для атома в матрице сопоставима с размером выделения, и в то же время низкая температура обеспечивает высокое отношение $D_{A}^{0} / D_{B}^{0}$, так что распад может реализоваться в условиях далеких от локального равновесия.

При низких температурах распад сплава реализуется с низкой скоростью, поэтому его экспериментальное наблюдение обычно оказывается возможным лишь при внешнем воздействии, которое само по себе может изменять кинетику превращения. Так в условиях низкотемпературной интенсивной пластической деформации, при которой скорость диффузии повышается на несколько порядков, выделения промежуточного состава наблюдались экспериментально в сплавах $\mathrm{Ag}-\mathrm{Cu}$ [16], $\mathrm{Fe}-\mathrm{Cu}[17]$. Однако в этих случаях объяснение связано скорее с эффектами прямого механического перемешивания [18] или с неравновесными диффузионными превращениями [19], тем более, что аналогичные дисперсные состояния в этих сплавах возникают при обработке смеси чистых компонентов.

В работах [20,21] приводились результаты 3D атом-проб томографии, свидетельствующие о выделениях ОЦК-меди промежуточного состава в матрице ОЦК-Fе на ранних стадиях распада при $T \sim 1000 \mathrm{~K}$. Однако результаты этих экспериментов противоречивы; по-видимому, эффект имеет место только в многокомпонентных сплавах. Исходя из таблицы, в ГЦК-решетке следовало бы ожидать скорее появления „рыхлых“ выделений железа в матрице меди, а не наоборот. Данные по коэффициентам диффузии примесей в объеме метастабильных выделений ОЦК-меди отсутствуют, что не позволяет указать предполагаемый состав выделений к моменту завершения волновой стадии распада в этом случае.

В работе [22] приведены результаты 3D-атом-проб томографии начальной стадии спинодального распада в сплаве $\mathrm{FeCr}$, содержащем около 30 at.\%. хрома. В этом случае выделения размером около $15 \mathrm{~nm}$ имеют состав в области промежуточных концентраций хрома ( 50 at.\%.) и неправильную форму, что кажется согласуется качественно с рис. 2.

\section{5. Заключение}

Предсказана возможность формирования выделений промежуточного состава, а также вторичных выделений внутри первичных, при спинодальном распаде в концентрированном твердом растворе в условиях достаточно низкой температуры, когда коэффициенты примесной диффузии компонентов существенно различаются.

\section{Финансирование работы}

Работа выполнена в рамках государственного задания по теме „Структура“ № AААА-А18-118020190116-6.

\section{Конфликт интересов}

Автор заявляет об отсутствии конфликта интересов.

\section{Список литературы}

[1] Дж. Кристиан. Теория превращений в металлах и сплавах. Мир, М. (1978). 806 c.

[2] J.W. Cahn, J.E. Hilliard. J. Chem. Phys. 28, 258 (1958).

[3] J.W. Cahn. Acta Met. 9, 795 (1961).

[4] J.W. Cahn. Trans. Met. Soc. AIME 242, 166 (1968).

[5] V.G. Vaks, S.V. Beiden, V.Yu. Dobretsov. Письма в ЖЭТФ 61, 65 (1995).

[6] V.G. Vaks. Phys. Rep. 391, 3-6, 157 (2004).

[7] I.M. Lifshits, V.V. Slyozov. J. Phys. Chem. Solids 19, 35 (1961). 
[8] A.D. Smigelskas, E.O. Kirkendall. Trans. Am. Inst. Min. (Met.) Engrs. 171, 130 (1947).

[9] Я.Е. Гегузин. Диффузионная зона. Наука, М. (1979). 344 с.

[10] В.Л. Гапонцев, И.К. Разумов, Ю.Н. Горностырев, А.Е. Ермаков, В.В. Кондратьев. ФММ 99, 4, 26 (2005).

[11] И.К. Разумов, Ю.Н. Горностырев. Влияние границ зерен на кинетику распада твердых растворов. В сб.: Науч. тр. IV шк.-семинара „Фазовые и структурные превращения в сталях“, Магнитогорск (2006). С. 99-112.

[12] I.K. Razumov, Yu.N. Gornostyrev, A.Ye. Yermakov. J. Alloys Compd 434-435, 535 (2007).

[13] Процессы взаимной диффузии в сплавах / Под ред. К.П. Гурова. Наука, М. (1973). 359 с.

[14] С.В. Терехов. ЖТФ 77, 8, 36 (2007).

[15] Landolt-Börnstein: Numerical Data and Functional Relationships in Science and Technology. New Series, Group III: Crystal and Solid State Physics. V. 26. Diffusion in Metals and Alloys / Ed. H. Mehrer. Springer-Verlag, Berlin (1990). 747 p.

[16] F. Wu, D. Isheim, P. Bellon, D.N. Seidman. Acta Mater. 54, 2605 (2006).

[17] N. Wanderka, U. Czubayko, V. Naundorf, V.A. Ivchenko, A.Ye. Yermakov, M.A. Uimin, H. Wollenberger. Ultramicroscopy 89, 189 (2001).

[18] P. Bellon, G. Martin. Phys. Rev. B 39, 4, 2403 (1989).

[19] И.К. Разумов, Ю.Н. Горностырев, А.Е. Ермаков. ФТТ 61, 2, 346 (2019).

[20] D. Isheim, M.S. Gagliano, M.E. Fine, D.N. Seidman. Acta Mater. 54, 841 (2006).

[21] M. Schober, E. Eidenberger, P. Staron, H. Leitner. Microsc. Microanal. 17, 26 (2011).

[22] W. Xiong, P. Hedstrom, M. Selleby, J. Odqvist, M. Thuvander, Q. Chen. CALPHAD 35, 355 (2011).

Редактор Ю.Э. Китаев 